\title{
Gamma-ray Spectroscopy
}

\author{
Jürgen Knödlseder \\ Centre d'Etude Spatiale des Rayonnements, 9, avenue du \\ Colonel-Roche, B.P. 4346, 31028 Toulouse Cedex, France
}

\begin{abstract}
Gamma-ray spectroscopy has made important progress with the observations of the OSSE and COMPTEL telescopes aboard CGRO, and has entered the domain of high-resolution spectroscopy with the launch of the SPI telescope aboard the INTEGRAL satellite. Recent science highlights in this field are presented, and an outlook is given to future promising projects.
\end{abstract}

\section{Introduction}

The gamma-ray domain around $\mathrm{MeV}$-energies is rich in emission lines that arise from transitions following nuclear de-excitation. Each isotope obeys its own characteristic gamma-ray line pattern, hence gamma-ray spectroscopy provides a powerful tool to analyse compositions and abundances. The opaqueness of the atmosphere to gamma-rays requires the use of space based telescopes or stratospheric balloons, with the unavoidable drawback of intense cosmic-ray bombardement that leads to an overwhelming instrumental background. Extracting the generally weak celestial signal from this background is the most challenging task in the analysis of gamma-ray spectroscopy data.

The richest harvest of gamma-ray spectroscopy results comes from the OSSE and COMPTEL telescopes aboard the Compton Gamma Ray Observatory (CGRO) satellite (observing period 1991-2000), although several small satellites and balloon telescopes have also provided significant contributions. Since October 2002, ESA's INTEGRAL satellite is in orbit, and provides with SPI a high-resolution imaging gamma-ray spectrometer with unprecedented performance. The Spectrometer on INTEGRAL (SPI) is equipped with 19 cooled germanium detector crystals that are arranged in a hexagonal pattern and act as the camera of the telescope. A coded mask situated 1.71 metres above the camera modulates the incoming gamma radiation, and provides an angular resolution of $\sim 2.5^{\circ}$ within a fully coded field of view of $16^{\circ}$. Typically, SPI reaches an energy resolution of $\sim 2.5 \mathrm{keV}$ at $1 \mathrm{MeV}$ with a narrow line sensitivity of $(2-3) \times 10^{-5} \mathrm{ph} \mathrm{cm}^{-2} \mathrm{~s}^{-1}$ for an observation time of $10^{6}$ seconds (Roques et al. 2003). The science objectives that will be addressed by SPI are the mapping of diffuse radioactivities to explore galactic nucleosynthetic activity, the search for the origin of galactic positrons, the study of supernova and nova nucleosynthesis, the search for nuclear excitation lines, and the study of cyclotron lines. In the following section, recent science highlights that have been achieved in these domains will be presented. 


\section{Science Highlights}

The $1.809 \mathrm{MeV}$ gamma-ray line of the radio-isotope ${ }^{26} \mathrm{Al}$ (half-life $7.2 \times 10^{5} \mathrm{yr}$ ) is so far the best studied non-solar gamma-ray line. COMPTEL observations provided the first all-sky map of the emission and clearly demonstrated that ongoing ${ }^{26} \mathrm{Al}$ nucleosynthesis is a galaxy-wide phenomenon (Oberlack et al. 1996). In particular, the gamma-ray line emission traces particularily well the distribution of the most massive stars in the Galaxy, hence it is believed that those are the nucleosynthetic sources of ${ }^{26} \mathrm{Al}$ (Knödlseder et al. 1999). Observations by the GRIS telescope (Naya et al. 1996) indicated a considerable broadening of the $1.809 \mathrm{MeV}$ line that theoretically was only poorly understood. New measurements using the RHESSI solar observatory (Smith 2003a) and SPI (Diehl et al. 2003), however, show that the $1.809 \mathrm{MeV}$ line is narrow, having a width that is compatible with the expected Doppler broadening that arises from the rotation of the Galaxy. Hence, high-resolution spectroscopy combined with imaging, such as provided by SPI, may allow measurements of the subtle line-shifts due to galactic rotation, and thus may provide kinematic distance information to galactic nucleosynthesis regions.

Recent RHESSI observations provided the first evidence for the detection of the 1.173 and $1.332 \mathrm{MeV}$ gamma-ray lines from the radioactive decay of ${ }^{60} \mathrm{Fe}$ (half-life $1.5 \times 10^{6} \mathrm{yr}$ ) in the general direction of the Galactic Centre (Smith 2003b). ${ }^{60} \mathrm{Fe}$ is believed to be mainly synthesised during supernovae explosions, hence a direct study of explosive nucleosynthesis becomes feasible (in contrast, ${ }^{26} \mathrm{Al}$ also has an important hydrostatic nucleosynthesis channel). Validating the RHESSI measurements and eventually mapping the distribution of the ${ }^{60} \mathrm{Fe}$ lines are major science objectives of the INTEGRAL mission.

The first gamma-ray line detected from the interstellar medium was the $511 \mathrm{keV}$ positron annihilation line (Johnson et al. 1972), yet the origin of the galactic positrons is still unclear. OSSE observations suggest at least two emission components: a prominent extended bulge, and a galactic disk. Hints of a third component, situated above the galactic disk, have gradually faded away with improvements of the data analysis techniques (Milne et al. 2003). Recent SPI observations clearly confirm the bulge emission, yet do not show any hint for a galactic disk component (Knödlseder et al. 2003). The acquired SPI exposure is probably not yet sufficient to detect the faint disk emission, and more data are needed to determine the $511 \mathrm{keV}$ emission distribution in detail. Yet once known, this distribution should provide information about the underlying sources, and hopefully unveil the origin of the positrons.

\section{Outlook}

The Holy Grail of gamma-ray line spectroscopy, the study of nucleosynthesis in nearby extragalactic supernovae, is still beyond the sensitivity limits of existing gamma-ray telescopes. A breakthrough could come from the usage of a gammaray lens, a concept that has been recently validated in a first series of balloon flights in France (Halloin et al. 2003). A gamma-ray lens makes use of Bragg reflection in appropriately designed and selected cristals, which if arranged in a ring, can focus gamma-rays from a large collection area onto a small focal 
spot. The relatively long focal length $(\sim 100 \mathrm{~m})$ requires a two satellite configuration in constellation, such as foreseen for other major astronomy missions (e.g., Darwin, XEUS, ...). Equipped with a small germanium detector, the lens telescope could reach sensitivities of $3 \times 10^{-7} \mathrm{ph} \mathrm{cm}^{-2} \mathrm{~s}^{-1}$ at $847 \mathrm{keV}$, allowing for line profile studies of supernovae nucleosynthesis products that will provide crucial information for the understanding of the explosion mechanism (Isern et al. 1997).

\section{References}

Diehl, R., Knödlseder, J., Lichti, G.G., et al. 2003, A\&A, 411, 451

Halloin, H., von Ballmoos, P., Evrard, J., et al. 2003, NIM-A, 504, 120

Isern, J., Gomez-Gomar, J., Bravo, E., \& Jean, P. 1997, in Proceedings 2nd INTEGRAL Workshop, St. Malo, France, eds. C. Winkler, T. Courvoisier, Ph. Durouchoux, ESA SP-382, 89

Johnson, W.N., Harnden, F.R., \& Haymes, R.C. 1972, ApJ, 172, L1

Knödlseder, J., Lonjou, V., Jean, P., et al. 2003, A\&A, 411, 457

Knödlseder, J., Bennett, K., Bloemen, H., et al. 1999, A\&A, 344, 68

Naya, J., Barthelmy, S.D., Bartlett, L.M., et al. 1996, Nature, 384, 44

Milne, P. et al. 2003, in Astronomy with Radioactivities IV, in press

Oberlack, U., Bennett, K., Bloemen, H., et al. 1996, A\&A, 120C, 311

Roques, J.-P., Schanne, S., von Kienlin, A., et al. 2003, A\&A, 411, 91

Smith, D. 2003a, ApJ, 589, 55

Smith, D. 2003b, in Astronomy with Radioactivities IV, in press 